\title{
Simulating a quantum magnet with trapped ions
}

\author{
A. FRIEDENAUER*, H. SCHMITZ*, J. T. GLUECKERT, D. PORRAS AND T. SCHAETZ ${ }^{\dagger}$ \\ Max-Planck-Institut für Quantenoptik, Hans-Kopfermann-Str. 1, D-85748 Garching, Germany \\ *These authors contributed equally to this work \\ †e-mail: tschaetz@mpq.mpg.de
}

To gain deeper insight into the dynamics of complex quantum systems we need a quantum leap in computer simulations. We cannot translate quantum behaviour arising from superposition states or entanglement efficiently into the classical language of conventional computers. The solution to this problem, proposed in 1982 (ref. 1), is simulating the quantum behaviour of interest in a different quantum system where the interactions can be controlled and the outcome detected sufficiently well. Here we study the building blocks for simulating quantum spin Hamiltonians with trapped ions ${ }^{2}$. We experimentally simulate the adiabatic evolution of the smallest non-trivial spin system from paramagnetic into ferromagnetic order with a quantum magnetization for two spins of $98 \%$. We prove that the transition is not driven by thermal fluctuations but is of quantum-mechanical origin (analogous to quantum fluctuations in quantum phase transitions ${ }^{3}$ ). We observe a final superposition state of the two degenerate spin configurations for the ferromagnetic order $(|\uparrow \uparrow\rangle+|\downarrow \downarrow\rangle)$, corresponding to deterministic entanglement achieved with $88 \%$ fidelity. This method should allow for scaling to a higher number of coupled spins ${ }^{2}$, enabling implementation of simulations that are intractable on conventional computers.

It is not possible to efficiently describe the time evolution of quantum systems on a classical device, such as a conventional computer, because their memory requirements grow exponentially with their size. For example, a classical memory would need to hold $2^{50}$ numbers to store arbitrary quantum states of 50 spin$1 / 2$ particles. The ability to calculate their evolution requires the derivation of a matrix of $\left(2^{50}\right)^{2}=2^{100}$ elements, already exceeding by far the capacity of state-of-the-art computers. Further, each doubling of computational power permits only one additional spin-1/2 particle to be simulated. To allow for deeper insight into quantum dynamics, we need a 'quantum leap' in simulation efficiency.

As proposed in ref. 1, a universal quantum computer would accomplish this step. A huge variety of possible systems are under investigation, with individual trapped ions ${ }^{4,5}$ as quantum bits (qubits) being a very promising architecture. After addressing the established criteria summarized in ref. 6 on up to eight ions ${ }^{7,8}$ with operational fidelities exceeding 99\% (refs 7-9), there seems to be no fundamental reason why such a device would not be realizable.

Alternatively, an analogue quantum computer, much closer to the original proposal in ref. 1, might allow for a shortcut towards quantum simulations. We would like to simulate a given system by a different one described by a Hamiltonian containing all the important features of the original system. The simulator system needs to be controlled, manipulated and measured in a sufficiently precise manner and has to be rich enough to address interesting questions about the original system. For large coupled spin systems, optical lattices might be advantageous ${ }^{10}$, whereas smaller spin systems and degenerate quantum gases might be simulated by trapped ions ${ }^{2,11}$. Instead of implementing a Hamiltonian with a universal set of gates, direct simulation of the Hamiltonian can be implemented by one (adiabatic) evolution of the initial state into the corresponding final state of interest.

Here, in a proof-of-principle experiment, we simulate the adiabatic transition from a quantum para- to a quantum (anti-) ferromagnet and illustrate the advantages of the adiabatic quantum simulation (Fig. 1). We demonstrate the individual access, via radio-frequency (r.f.) and laser fields, to all relevant parameters in the underlying Hamiltonian, representing one out of a large spectrum of quantum spin Hamiltonians.

The adiabatic quantum simulation of generic spin Hamiltonians proposed in ref. 2 can be illustrated considering a string of charged spin-1/2 particles confined in a common harmonic potential. Two electronic states of each ion simulate the two-level system of a spin- $1 / 2$ magnetic moment, $|\uparrow\rangle$ and $|\downarrow\rangle$. The inter-ion distance of several micrometres renders any direct spin-spin coupling negligible. The quantum Ising Hamiltonian,

$$
H_{\text {Ising }}=H_{B}+H_{J}=-B_{x} \sum_{i} \sigma_{i}^{x}+\sum_{i<j} J_{i j} \sigma_{i}^{z} \sigma_{j}^{z},
$$

consists of two terms. The first denotes the interaction of each individual spin, represented by the Pauli operator $\sigma_{i}^{k}$ ( $k$ can be $x, y$ or $z$ ), with a uniform magnetic field of amplitude proportional to $B_{x}$ pointing in direction $x$. The second term represents the spin-spin interaction, which tries to align the spins $\left(\sigma_{i}^{z}\right)$ parallel or antiparallel along the $z$ axis, depending on the sign of the interaction amplitude $J_{i j}$. To simulate the first term in equation (1), the eigenstates of $\sigma_{i}^{z},|\uparrow\rangle_{i}$ and $|\downarrow\rangle_{i}$, can be coupled with an electromagnetic field. The latter is simulated by a state-dependent force $^{12}$, further explained in Fig. 2.

To understand the experiment discussed below, we consider interactions between nearest neighbours only, $J_{i, i+1}=J$, and two extreme scenarios. For the case of $J=0$ and $B_{x}>0$, the ground state of the spin system has all spins aligned with $B_{x}$ along the $x$ axis. This corresponds to the paramagnetically ordered state $|\rightarrow \rightarrow \ldots \rightarrow\rangle$, the eigenstate of the Hamiltonian $H_{B}=-B_{x} \sum_{i} \sigma_{i}^{x}$ with the lowest energy. 


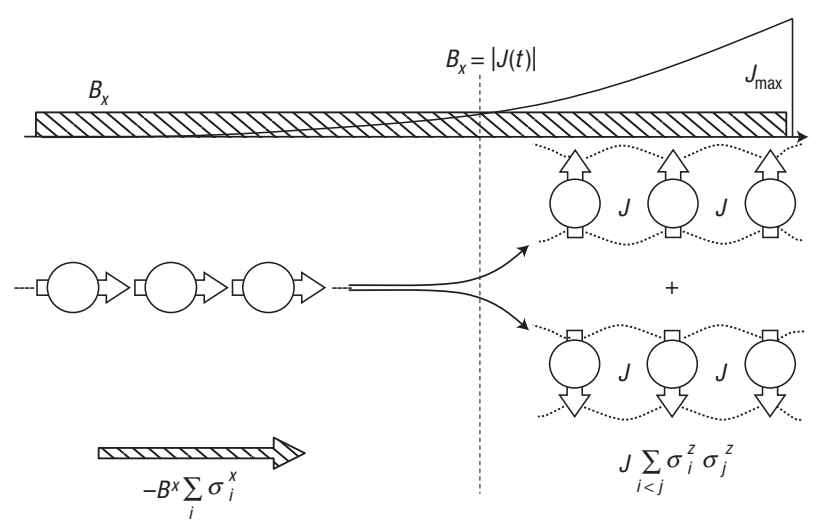

Figure 1 Phase transition of a quantum magnet. Each ion can simulate a magnetic spin, analogous to an elementary magnet. We initialize the $N$ spins in the paramagnetic state $|\rightarrow \rightarrow \ldots \rightarrow\rangle$, the ground state of the Hamiltonian $H_{B}=-B_{x}\left(\sigma_{1}^{x}+\sigma_{2}^{x}+\cdots+\sigma_{N}^{x}\right)$. This is equivalent to aligning the spins parallel to the simulated magnetic field. Adding an effective spin-spin interaction $J(t)$ (at constant $B_{x}$ ) and increasing it adiabatically to $\left|J_{\max }\right| \gg B_{x}$, we expect the system to undergo a quantum phase transition into a ferromagnet, the new ground state of the system ( $J$ is symbolized as short chains, trying to keep neighbouring spins aligned). Ideally, the two possible ferromagnetic orders $|\uparrow \uparrow \ldots \uparrow\rangle$ and $|\downarrow \downarrow \ldots \downarrow\rangle$ are degenerate ground states. The spin system should evolve into the superposition state $|\uparrow \uparrow \ldots \uparrow\rangle+|\downarrow \downarrow \ldots \downarrow\rangle$, a maximally entangled 'Schrödinger cat' state/magnet.

For the opposite case of $B_{x}=0$ and $J<(>) 0$, the system has an infinite number of degenerate ground states, defined by any superposition of the lowest-energy eigenstates of $\sum_{i<j} J \sigma_{i}^{z} \sigma_{i}^{z}$, namely $|\uparrow \uparrow \ldots \uparrow\rangle$ and $|\downarrow \downarrow \ldots \downarrow\rangle$, which represent ferromagnetic order (or $|\uparrow \downarrow \uparrow \ldots \uparrow \downarrow\rangle$ and $|\downarrow \uparrow \downarrow \ldots \downarrow \uparrow\rangle$, the antiferromagnetic order, respectively). Initializing the spin system in an eigenstate in the $\sigma^{x}$ basis, starting with $J(t=0)=0, B_{x}>0$ and adiabatically increasing $|J(t)|$ to $\left|J_{\max }\right| \gg B_{x}$ should evolve the system from the paramagnetic order arbitrarily close to the (anti-) ferromagnetic order, as depicted in Fig. 1. A quantum phase transition is supposed to occur at $B_{x}=|J|$ in the thermodynamic limit of an infinite number of spins ${ }^{3,13}$.

We experimentally demonstrate the above features on two spins as follows. We confine two ${ }^{25} \mathrm{Mg}^{+}$ions in a linear Paul trap ${ }^{14}$ and laser-cool them to the Coulomb-crystalline phase, where the ions align along the trap axis $a$. The motion of the ions along this axis $a$ can be described in the basis of normal modes. The in-phase mode oscillates at a frequency $\omega_{\text {com }} / 2 \pi=2.1 \mathrm{MHz}$, whereas the out-of-phase mode has $\omega_{\text {stretch }} / 2 \pi=\sqrt{3} \omega_{\text {com }} / 2 \pi=3.7 \mathrm{MHz}$.

In our implementations we define the ${ }^{2} S_{1 / 2}$ hyperfine ground states as $|\downarrow\rangle \equiv\left|F=3 ; m_{f}=3\right\rangle$ and $|\uparrow\rangle \equiv\left|F=2 ; m_{f}=2\right\rangle$, which are separated by $\omega_{0} / 2 \pi \simeq 1.7 \mathrm{GHz}$. An external magnetic field $B$ (different from the simulated magnetic field in equation (1)) of $5.5 \mathrm{G}$ orients the magnetization axis for the projection $\hbar m_{f}$ of each ion's angular momentum $F$. In this field adjacent Zeeman sublevels of the $F=3$ and 2 manifolds are split by $2.7 \mathrm{MHz}$ per level.

We coherently couple the states $|\downarrow\rangle$ and $|\uparrow\rangle$ with a resonant r.f. field at $\omega_{0}$ to implement single spin rotations ${ }^{14-16}$

$$
R(\Theta, \phi)=\cos (\Theta / 2) I-i \sin (\Theta / 2) \cos \phi \sigma^{x}-i \sin (\Theta / 2) \sin \phi \sigma^{y},
$$

where $I$ is the identity operator, $\sigma^{x}$ and $\sigma^{y}$ denote the Pauli spin matrices acting on $|\uparrow\rangle_{i}$ and $|\downarrow\rangle_{i}, \Theta / 2=B_{x} t / \hbar$ is proportional to
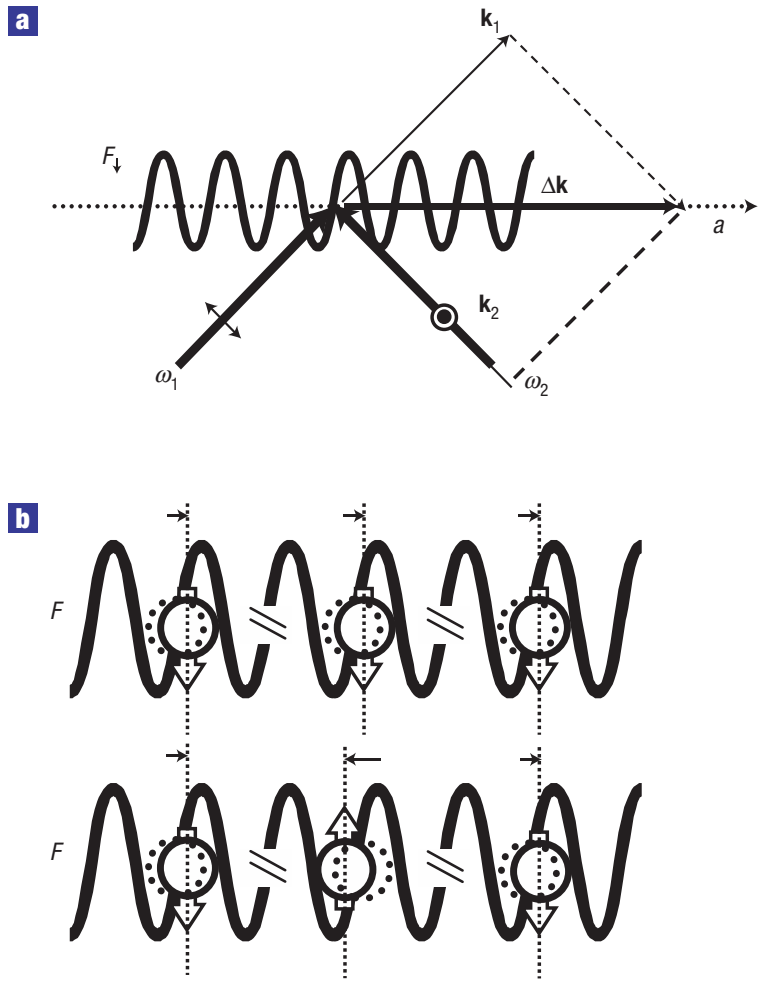

Figure 2 Simulating the quantum magnet. a, Two perpendicular polarized laser beams of frequencies $\omega_{1}$ and $\omega_{2}$ induce a state-dependent optical-dipole force $F_{\downarrow}=-(3 / 2) F_{\uparrow}$ by the a.c. Stark shift (here, only $F_{\downarrow}$ is depicted). The wavevector difference $\Delta \mathbf{k}=\mathbf{k}_{2}-\mathbf{k}_{1}$ points along the trap axis $a$. $\mathbf{b}$, For a standing wave $\omega_{1}=\omega_{2}$, the force conditionally changes the distance between neighbouring spins, simulating a spin-spin interaction ${ }^{2}\left(F_{\uparrow}\left(F_{\downarrow}\right)\right.$ symbolized by the arrow to the right (left)). Only if all spins are aligned (top) does the distance between the spins (that is, between the ions) remain unchanged. Therefore, the total Coulomb energy of the spin system is not increased, defining ferromagnetic order, the quantum magnet, to be the ground state. For $\omega_{1} \neq \omega_{2}$, the sinusoidal force pattern can be seen as a wave moving along the trap axis a pushing or pulling the ions repeatedly at a frequency $\omega_{1}-\omega_{2}$. We chose $\omega_{1}-\omega_{2}$ close to the resonance frequency of the ions oscillating out of phase (stretch mode). The energies of different spin chains now depend on the coupling of the spin state to the stretch mode. Energy can be coupled efficiently into the state with different spin orientations (bottom, for example), defining the unaffected upper case as a ground state ${ }^{18}$. The interpretation in terms of an effective spin-spin interaction is further described in the Methods section.

the duration $t$ of the rotation and $\phi$ is the phase of the r.f. oscillation defining the axis of rotation in the $x-y$ plane of the Bloch sphere ${ }^{15}$.

We provide the effective spin-spin interaction by a state-dependent optical-dipole force ${ }^{12,16,17}$. The relative amplitudes $F_{\downarrow}=-(3 / 2) F_{\uparrow}$ are due to a.c. Stark shifts induced by two laser beams at a wavelength $\lambda$ of $280 \mathrm{~nm}$, depicted in Fig. 2a, perpendicular in direction and polarization, with their wavevector difference pointing along the trap axis $a$. They are detuned $80 \mathrm{GHz}$ blue of the ${ }^{2} P_{3 / 2}$ excited state, with intensities allowing for $|J / \hbar|$ as large as $2 \pi \times 22.1 \mathrm{kHz}$. We use a walking-wave force-pattern by detuning the two laser frequencies by $2 \pi \times 3.45 \mathrm{MHz}=\omega_{\text {stretch }}+\delta$, with $\delta=-2 \pi \times 250 \mathrm{kHz}$. This choice avoids several technical problems of the original proposal ${ }^{2}$ (see the Methods section), while at the same time resonantly enhancing the effective spin-spin interaction by a factor of $\left|\omega_{\text {stretch }} / \delta\right|=14.8$ compared with the 


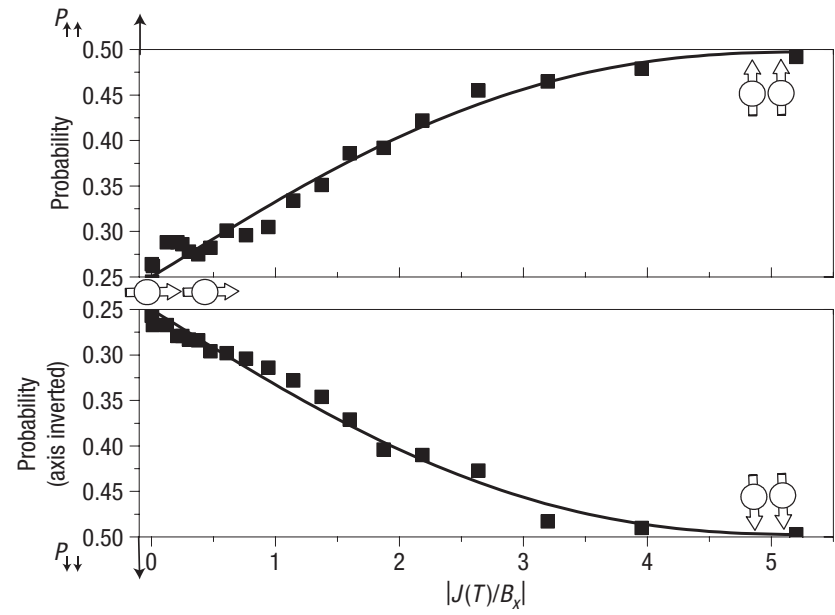

Figure 3 Quantum magnetization of the spin system. We initialize the spins in the paramagnetic state $|\rightarrow\rangle|\rightarrow\rangle \equiv(|\uparrow\rangle+|\downarrow\rangle)(|\uparrow\rangle+|\downarrow\rangle)=|\uparrow \uparrow\rangle+|\downarrow \uparrow\rangle+$ $|\uparrow \downarrow\rangle+|\downarrow \downarrow\rangle$, the ground state of the Hamiltonian $H_{B}=-B_{x}\left(\sigma_{1}^{x}+\sigma_{2}^{x}\right)$. A measurement of this superposition state will already project into both $|\uparrow \uparrow\rangle$ and $|\downarrow \downarrow\rangle$, each with a probability of 0.25 . After applying $B_{x}$ we adiabatically increase the effective spin-spin interaction from $J(t=0)=0$ to $J(T)$. State-sensitive fluorescence detection enables us to distinguish the final states $|\uparrow \uparrow\rangle,|\downarrow \downarrow\rangle,|\uparrow \downarrow\rangle$ or $|\downarrow \uparrow\rangle$. Averaging over $10^{4}$ experiments provides us with the probability distributions $P_{\downarrow \downarrow}$ (two ions fluoresce), $P_{\uparrow \uparrow}$ (no ion fluoresces) and $P_{\uparrow \downarrow}$ or $P_{\downarrow \uparrow}$ (one ion fluoresces). We repeat the measurement for increasing ratios $\left|J(T) / B_{x}\right|$. The experimental results for the two ferromagnetic contributions $P_{\downarrow \downarrow}$ and $P_{\uparrow \uparrow}$ are depicted as squares. Solid lines are the result of a calculation of the evolution of the effective spin system induced by $B_{x}$ and $J(t)$. For $\left|J(T) / B_{x}\right| \ll 1$, the paramagnetic order is preserved. For $\left|J(T) / B_{x}\right| \gg 1$, the spins undergo a transition into the ferromagnetic order, the ground state of the Hamiltonian $H_{J}=J_{\max } \sigma_{1}^{z} \sigma_{2}^{z}$, with a related quantum magnetization $M=P_{\downarrow \downarrow}+P_{\uparrow \uparrow} \geq 98 \pm 2 \%$. Note that we invert the ordinate of the lower frame to emphasize the unbroken symmetry of the evolution.

standing-wave case ${ }^{18}$. The enhancement is induced by the fact that the walking wave is closer to resonance (detuned only by $\delta$ ) with the vibrational mode compared with the standing wave (detuned by $\left.\omega_{\text {stretch }}\right)$.

After laser cooling we initialize the quantum simulator by optical pumping ${ }^{19}$ to the state $|\downarrow\rangle|\downarrow\rangle|n \simeq 0\rangle$. We rotate both spins in a superposition state via an $R(\pi / 2,-\pi / 2)$ pulse (see equation (2)) on the r.f. transition to initialize the state $\left|\Psi_{i}\right\rangle=|\rightarrow\rangle|\rightarrow\rangle|n \simeq 0\rangle$. Note that the paramagnetic state $|\rightarrow\rangle|\rightarrow\rangle \equiv(|\uparrow\rangle+|\downarrow\rangle)(|\uparrow\rangle+$ $|\downarrow\rangle)=|\uparrow \uparrow\rangle+|\downarrow \uparrow\rangle+|\uparrow \downarrow\rangle+|\downarrow \downarrow\rangle$ has a $25 \%$ probability to be projected into either $|\uparrow \uparrow\rangle$ or $|\downarrow \downarrow\rangle$ (normalization factors are suppressed throughout).

We simulate the effective magnetic field by continuously applying an r.f. field with phase $\phi=0$ and an amplitude such that it corresponds to a single qubit rotation $R(\Theta, 0)$ with full rotation period $\Theta=2 \pi$ in $118 \mu$ s and deduce $B_{x} / \hbar=2 \pi \times 4.24 \mathrm{kHz}$. Precise control of the phase $\phi$ of the r.f. oscillator relative to the initialization pulse enables alignment of $B_{x}$ parallel to the spins along the $x$ axis in the equatorial plane of the Bloch sphere, ensuring that $\left|\Psi_{i}\right\rangle$ is an eigenstate of this effective magnetic field.

At the same time, we switch on the effective spin-spin interaction $J(t)(t \in[0 ; T])$ and increase its amplitude adiabatically up to $J(T)$ within 50 steps of $2.5 \mu$ s each. At time $T$, we switch off the interactions and analyse the final state of the two spins via the state-sensitive detection described below. In a sequence of experiments at constant $B_{x}$ we increase $J_{\max }$ and

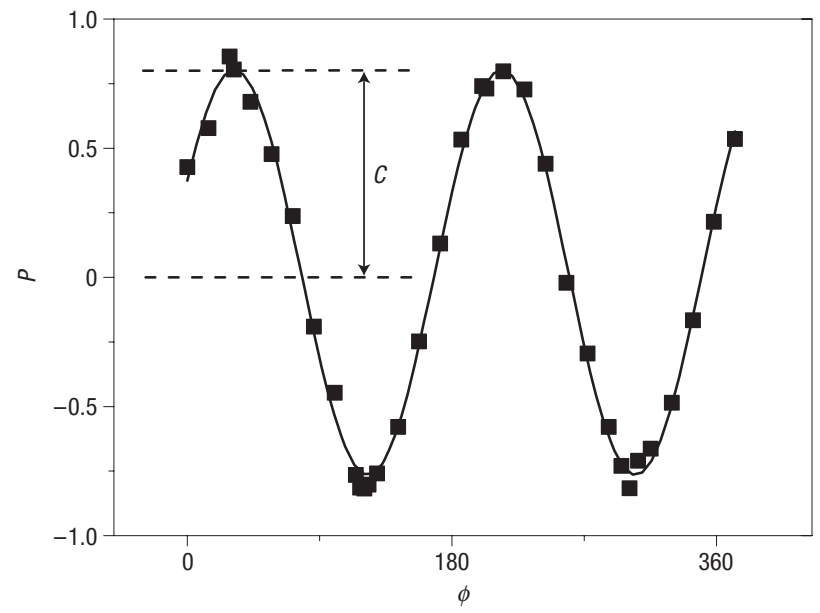

Figure 4 Entanglement of the quantum magnet. Measurement of the parity $P=P_{\downarrow \downarrow}+P_{\uparrow \uparrow}-\left(P_{\downarrow \uparrow}+P_{\uparrow \downarrow}\right)$ of the final ferromagnetic state after the simulation reached $\left|J_{\max } / B_{x}\right|=5$.2. As we vary the phase $\phi$ of a subsequent analysis pulse $R(\pi / 2, \phi)$, the parity of the two spins oscillates as $C \cos (2 \phi)$. Together with the final-state populations $P_{\downarrow \downarrow}$ and $P_{\uparrow \uparrow}$ depicted in Fig. 3, we can deduce the fidelity $F=\left|\left\langle\Psi_{\text {final }} \mid \downarrow \downarrow+\uparrow \uparrow\right\rangle\right|^{2}=1 / 2\left(P_{\downarrow \downarrow}+P_{\uparrow \uparrow}\right)+C / 2=88 \pm 3 \%$ for the final superposition state $|\uparrow \uparrow\rangle+|\downarrow \downarrow\rangle$, a maximally entangled state, highlighting the quantum nature of this transition. We find qualitatively comparable results for the antiferromagnetic case $|\uparrow \downarrow\rangle+|\downarrow \uparrow\rangle$. Each data point represents an average over $10^{4}$ experiments.

therefore $\left|J(T) / B_{x}\right|$. Finally we reach the maximal amplitude $\left|J(t=125 \mu \mathrm{s}) / B_{x}\right|=\left|J_{\max } / B_{x}\right|=5.2$ (see the Methods section) and achieve a quantum magnetization $M$, the probability of being in a state with ferromagnetic order, of $M=P_{\downarrow \downarrow}+P_{\uparrow \uparrow}=98 \% \pm 2 \%$.

After the adiabatic evolution described above, we project the final spin state into the $\sigma^{z}$ measurement basis by a laser beam tuned resonantly to the $|\downarrow\rangle \leftrightarrow{ }^{2} P_{3 / 2}\left|F=4 ; m_{f}=4\right\rangle$ cycling transition ${ }^{16}$. An ion in state $|\downarrow\rangle$ fluoresces brightly, leading to the detection of an average of 40 photons during a $160 \mu$ s detection period with our photomultiplier tube. In contrast, an ion in state $|\uparrow\rangle$ remains close to dark (on average six photons). We repeat each experiment for the same set of parameters $10^{4}$ times and derive the probabilities $P_{\downarrow \downarrow}, P_{\uparrow \uparrow}$ and $P_{\downarrow \uparrow}$ for the final state being projected into state $|\downarrow \downarrow\rangle$, $|\uparrow \uparrow\rangle$ and $(|\downarrow \uparrow\rangle$ or $|\uparrow \downarrow\rangle)$, respectively (further described in the Methods section).

In our experiment we can detect both ferromagnetic contributions, $P_{\uparrow \uparrow}$ and $P_{\downarrow \downarrow}$, separately. Any imperfection in the simulation acting as a bias field $B_{z}$ along the $z$ axis would energetically prefer one of the ferromagnetic states over the other and therefore unbalance their contributions to the final state. We carefully cancel all bias fields (see the Methods section), to balance the populations $P_{\uparrow \uparrow}$ and $P_{\downarrow \downarrow}$ as can be seen in Fig. 3 . The results are in good agreement with theoretical predictions for our experiment, shown as solid lines. We expect the final state to be a coherent superposition of the two ferromagnetic states $|\uparrow \uparrow\rangle+|\downarrow \downarrow\rangle-$ a maximally entangled Bell state. To quantify the experimentally reached coherence we measure the parity ${ }^{20}$ $P=P_{\downarrow \downarrow}+P_{\uparrow \uparrow}-\left(P_{\downarrow \uparrow}+P_{\uparrow \downarrow}\right)$ after applying an additional $R(\pi / 2, \phi)$ pulse to both ions after $J_{\max }$ is reached, with a variable r.f. phase $\phi$ relative to the r.f. field simulating $B_{x}$. The measured data shown in Fig. 4 have a component that oscillates as $C \cos (2 \phi)$, where $|C| / 2$ characterizes the coherences between the $|\uparrow \uparrow\rangle$ and the $|\downarrow \downarrow\rangle$ components in the final state $\left|\Psi_{\text {final }}\right\rangle$ produced. Deducing a contrast 
$C$ of $78 \pm 2 \%$ from the best fit we derive the Bell-state fidelity ${ }^{20}$ $F=\left|\left\langle\Psi_{\text {final }} \mid \downarrow \downarrow+\uparrow \uparrow\right\rangle\right|^{2}=1 / 2\left(P_{\downarrow \downarrow}+P_{\uparrow \uparrow}\right)+C / 2$ of $88 \pm 3 \%$.

We also simulate the adiabatic evolution of a system not initialized in the ground state of the initial Hamiltonian. In particular, we prepare the paramagnetic eigenstate $|\leftarrow \leftarrow\rangle=(|\downarrow\rangle-|\uparrow\rangle)(|\downarrow\rangle-|\uparrow\rangle)$, with the spins aligned antiparallel with respect to the simulated magnetic field via an $R(\pi / 2, \pi / 2)$ r.f. initialization pulse. The adiabatic evolution should preserve the spin system in its excited state, leading now into the antiferromagnetic order $|\uparrow \downarrow\rangle+|\downarrow \uparrow\rangle$. After evolution to $J=J_{\max }$ we find $P_{\downarrow \uparrow}+P_{\uparrow \downarrow} \geq 95 \pm 2 \%$. To investigate the coherence between the $|\uparrow \downarrow\rangle$ and the $|\downarrow \uparrow\rangle$ components, we first rotate the state via an additional $R(\pi / 2,0)$ pulse, which would ideally take $|\uparrow \downarrow\rangle+|\downarrow \uparrow\rangle \rightarrow|\uparrow \uparrow\rangle+|\downarrow \downarrow\rangle$, followed by the measurement of the parity as explained above. We deduce the fidelity of the antiferromagnetic entangled state $F=\left|\left\langle\Psi_{\text {final }} \mid \downarrow \uparrow+\uparrow \downarrow\right\rangle\right|^{2}=1 / 2\left(P_{\downarrow \uparrow}+P_{\uparrow \downarrow}\right)+C / 2=80 \pm 4 \%$.

An equally valid viewpoint of this experiment interprets $|\leftarrow \leftarrow\rangle$ as the ground state of the Hamiltonian $-H_{\text {Ising. }}$. Because the sign of all spin-spin interactions is also reversed in $-H_{\text {Ising, }}$, it is equivalent to a change of sign in the spin-spin interaction $J$.

The entanglement of the final states confirms that the transition from paramagnetic to (anti-) ferromagnetic order is not caused by thermal fluctuations driving thermal phase transitions. The evolution is coherent and quantum mechanical, the coherent equivalent to the so-called quantum fluctuations $s^{3,13}$ driving quantum phase transitions in the thermodynamic limit. In this picture, tunnelling processes ${ }^{13}$ induced by $B_{x}$ couple the degenerate (in the rotating frame) states $|\uparrow\rangle_{i}$ and $|\downarrow\rangle_{i}$ with an amplitude proportional to $\left(\left|B_{x} / J\right|\right)$. In a simplified picture for $N$ spins, the amplitude for the tunnelling process between $\Psi_{N \uparrow}=|\uparrow \uparrow \ldots \uparrow\rangle$ and $\Psi_{N \downarrow}=|\downarrow \downarrow \ldots \downarrow\rangle$ is proportional to $\left(\left|B_{x} / J\right|\right)^{N}$, because all $N$ spins must be flipped. In the thermodynamic limit $(N \rightarrow \infty)$, the system is predicted to undergo a quantum phase transition at $|J|=B_{x}$. At values $|J|>B_{x}$, the tunnelling between $\Psi_{\infty \uparrow}$ and $\Psi_{\infty \downarrow}$ is completely suppressed. In our case of a finite system, $\Psi_{2 \uparrow}$ and $\Psi_{2 \downarrow}$ remain coupled and the sharp quantum phase transition is smoothed into a gradual change from paramagnetic to (anti-) ferromagnetic order.

In conclusion, we have demonstrated the feasibility of simple quantum simulations in an ion trap by implementing the Hamiltonian of a quantum magnet undergoing a robust transition from a paramagnetic to an entangled ferromagnetic or antiferromagnetic order. Although our system is currently too small to solve classically intractable problems, it uses an approach that is complementary to a universal quantum computer in a way that can become advantageous as the approach is scaled to larger systems. Our scheme does not rely on the use of sequences of quantum gates, thus scaling to a higher number of ions can be simpler, because it only requires inducing the same overall spin-dependent optical force on all the ions ${ }^{2}$. Furthermore, the desired outcome might not be affected by decoherence as drastically as typical quantum algorithms, because a continuous loss of quantum fidelity might not completely spoil the outcome of the experiment.

For example, the coherence of a Greenberger-Horne-Zeilingerlike state $|\uparrow \uparrow \ldots \uparrow\rangle+|\downarrow \downarrow \ldots \downarrow\rangle$ might be completely lost, but still the output of the quantum simulation describes correctly the ground state in a solid-state system, where spontaneous symmetry breaking implies ferromagnetic ordering randomly along one of two antiparallel orientations. In contrast, universal quantum computation will almost certainly require involved subalgorithms for error correction ${ }^{5}$. Decoherence in the simulator might even mimic the influence of the natural environment ${ }^{21}$ of the studied system if we judiciously construct our simulation (for example, the decoherence we mainly observe in our demonstration implements a dephasing environment).
Despite technical challenges, we expect that this work is the start to extensive experimental research of complex many-body phases with trapped-ion systems. Linear trapping set-ups may be used for the quantum simulation of quantum dynamics beyond the ground state, where chains of 30 spins would already enable us to outperform current simulations with classical computers. We may also adapt our scheme to new ion-trapping technologies ${ }^{22}$. For example, a modest scaling to systems of $20 \times 20$ spins in two dimensions would yield insight into open problems in solid-state physics, for example related to spin frustration. This could pave the way to address a broad range of fundamental issues in condensedmatter physics that are intractable with exact numerical methods, such as spin liquids in triangular lattices, suspected to be closely related to phases of high-temperature superconductors ${ }^{23}$.

\section{METHODS}

\section{STATE-DEPENDENT OPTICAL-DIPOLE FORCE}

An effective (Ising) spin-spin interaction was proposed to be implemented via magnetic field gradients ${ }^{24}$. It was suggested to use state-dependent opticaldipole forces ${ }^{2,11,17}$ displacing the spin state $|S\rangle(S$ either $\downarrow$ or $\uparrow)$ in phase space by an amount that depends on $|S\rangle$. The area swept in phase space changes the state to $\mathrm{e}^{i \phi(S)}|S\rangle$. The phase $\phi(S)$ can be broken down into single-spin terms proportional to $\sigma_{i}^{z}$ and apparent spin-spin interactions proportional to $\sigma_{i}^{k} \sigma_{j}^{k}$ and thus gives rise to the desired simulation of spin-spin interactions ${ }^{25}$. It can also lead to single-spin phases that simulate the unwanted contribution of a common bias field $B_{z} \sigma^{z}$ in the Hamiltonian that will result in an imbalance of the probabilities $P_{\downarrow \downarrow}$ and $P_{\uparrow \uparrow}$. To achieve a balanced probability distribution as depicted in Fig. 3, we have to carefully compensate these single-spin phases. To this end we compensate the residual a.c. Stark shifts of the individual laser beams by carefully choosing the direction and polarization of the beams ${ }^{12}$ and also compensate for the imbalance caused by single-spin phases via a detuning of the order of several kilohertz of the r.f. transition relative to $\omega_{0}$. Furthermore, the ions have to be separated by an integer multiple of the effective wavelength $\lambda_{\text {eff }}=\lambda / \sqrt{2}$; in our implementation $18 \lambda_{\text {eff }} \approx 3.6 \mu \mathrm{m}$, requiring the control of the axial trapping frequency to better than $100 \mathrm{~Hz}$.

To minimize the errors of our simulation, we have to keep the motional excitation small enough during the evolution to enable the system to be described within the Lamb-Dicke regime ${ }^{16}$ (ideally $\bar{n} \times \eta^{2} \ll 1$ ). The Lamb-Dicke factor $\eta$ can be interpreted as the ratio between the width of the ground-state wavefunction of the ion and $\lambda_{\text {eff }} / 2 \pi$ (for our parameters $\eta \approx 0.25$ ).

In addition, we have to return the system close to its motional ground state at the end of the simulation to minimize the errors due to residual spin-motion coupling $^{2}$ causing entanglement between the spin states and the motional degrees of freedom. To fulfil the first condition, we detune the two laser beams far enough from resonantly exciting the motional modes. Adjusting the detuning $\delta=-\left(\omega_{\text {stretch }}-\left(\omega_{1}-\omega_{2}\right)\right)=-2 \pi \times 250 \mathrm{kHz}$ red of the stretch-mode frequency reduces the motional excitation during one step of the evolution and on average to $\bar{n} \approx 1$. Finally terminating the evolution after the system returned into its motional ground state ${ }^{17}$, it ideally completely cancels the simulation errors discussed in ref. 2, allowing for the measured contrast of the parity oscillations depicted in Fig. 4.

\section{STATE-SENSITIVE DETECTION}

For two spins, the integrated fluorescence signal does not enable us to distinguish between two $(|\downarrow \uparrow\rangle$ and $|\uparrow \downarrow\rangle)$ of the four possible spin configurations. In addition, the number of detected photons for each of the three distinguishable configurations fluctuates from experiment to experiment according to Poissonian statistics and therefore can be determined only with limited accuracy. For the data reported, we repeated each experiment $10^{4}$ times and fitted the resulting photon-number distribution to the weighted sum of three reference distributions to derive $P_{\downarrow \downarrow}, P_{\uparrow \uparrow}$ and $P_{\downarrow \uparrow}+P_{\uparrow \downarrow}$.

\section{ADIABATIC EVOLUTION}

We achieve the best fidelities for the reported transitions at a duration of the simulation of $T=125 \mu$ s at a $B_{x}$ of $2 \pi \times 4.24 \mathrm{kHz}$. We are not strictly in the adiabatic limit, where for $T \rightarrow \infty$ the final ratio $\left|J_{\max } / B_{x}\right|=5.2$ allows for a maximum quantum magnetization of $93.4 \%$. The robustness of the coupling scheme used enables us to minimize decoherence effects and even to 
enhance the final quantum magnetization to $98 \%$ by reducing the duration of the simulation.

In addition, technical factors related to the specific nonlinear performance of the r.f. attenuator used to control $J(t)$ led to its evolution being linear in time to up to $J(t=50 \mu \mathrm{s})=5 \times 10^{-4} J_{\max }$, continued by $J(t) \sim\left(\mathrm{e}^{\alpha t}-\beta\right)^{2}$ best fitted by $\alpha=26 \times 10^{3} \mathrm{~s}^{-1}$ and $\beta=4$. So far we have not improved the fidelities by evolving or terminating $J(t)$ or $B_{x}$ in a more adiabatic way.

Received 27 March 2008; accepted 25 June 2008; published 27 July 2008.

\section{References}

1. Feynman, R. P. Simulating physics with computers. Int. J. Theor. Phys. 21, 467-488 (1982).

2. Porras, D. \& Cirac, J. I. Effective quantum spin systems with trapped ions. Phys. Rev. Lett. 92, 207901 (2004)

3. Sachdev, S. Quantum Phase Transitions (Cambridge Univ. Press, Cambridge, 1999).

4. http://qist.lanl.gov/qcomp_map.shtml.

5. Nielsen, M. \& Chuang, I. Quantum Computation and Quantum Information (Cambridge Univ. Press, Cambridge, 2000).

6. DiVincenzo, D. P. in Scalable Quantum Computation (eds Braunstein, S. L., Lo, H. K. \& Kok, P.) 1-13 (Wiley, New York, 2001).

7. Leibfried, D. et al. Creation of a six-atom 'Schrödinger cat' state. Nature 438, 639-642 (2005).

8. Häffner, H. et al. Scalable multiparticle entanglement of trapped ions. Nature 438, 643-646 (2005).

9. Benhelm, J., Kirchmair, G., Roos, C. F. \& Blatt, R. Towards fault-tolerant quantum computing with trapped ions. Nature Phys. 4, 463-466 (2008).

10. Greiner, M., Mandel, O., Esslinger, T., Haensch, T. W. \& Bloch, I. Quantum phase transition from a superfluid to a Mott insulator in a gas of ultracold atoms. Nature 415, 39-44 (2002).

11. Porras, D. \& Cirac, J. I. Bose-Einstein condensation and strong-correlation behaviour of phonons in ion traps. Phys. Rev. Lett. 93, 263602 (2004).

12. Wineland, D. J. et al. Quantum information processing with trapped ions. Phil. Trans. R. Soc. Lond. A 361, 1349-1361 (2003)
13. Sachdev, S. Quantum criticality: Competing ground states in low dimensions. Science 288, $475-480$ (2000).

14. Schaetz, T., Friedenauer, A., Schmitz, H., Petersen, L. \& Kahra, S. Towards (scalable) quantum simulations in ion traps. J. Mod. Opt. 54, 2317-2325 (2007).

15. Allen, L. \& Eberly, J. H. Optical Resonance and Two-Level Atoms (Dover, New York, 1987).

16. Wineland, D. J. et al. Experimental issues in coherent quantum-state manipulation of trapped atomic ions. J. Res. Natl Inst. Stand. Technol. 103, 259-328 (1998).

17. Leibfried, D. et al. Experimental demonstration of a robust, high fidelity geometric two ion-qubit phase gate. Nature 422, 412-415 (2003).

18. Porras, D. \& Cirac, J. I. Quantum manipulation of trapped ions in two dimensional Coulomb crystals. Preprint at $<$ http://arxiv.org/abs/quant-ph/0601148v3> (2006).

19. King, B. E. et al. Cooling the collective motion of trapped ions to initialize a quantum register. Phys. Rev. Lett. 81, 1525-1528 (1998).

20. Sackett, C. A. et al. Experimental entanglement of four particles. Nature 404, 256-259 (2000).

21. Lloyd, S. Universal quantum simulators. Science 273, 1073-1078 (1996).

22. Chiaverini, J. \& Lybarger, W. E. Jr. Laserless trapped-ion quantum simulations without spontaneous scattering using microtrap arrays. Phys. Rev. A 77, 022324 (2008).

23. Orestein, J. \& Millis, A. J. Advances in the physics of high- $T_{\mathrm{c}}$ superconductivity. Science 288, $468-474(2000)$.

24. Wunderlich, C. Laser Physics at the Limit 261-273 (Springer, Heidelberg, 2002)

25. Wang, X, Sorensen, K. \& Molmer, A. Multibit gates for quantum computing. Phys. Rev. Lett. 86, 3907-3910 (2001).

\section{Acknowledgements}

This work was supported by the Emmy-Noether Programme of the German Research Foundation (DFG, grant No SCHA 973/1-2) the MPQ Garching, the DFG Cluster of Excellence Munich-Centre for Advanced Photonics and the European project SCALA. We thank D. Leibfried for his input and I. Cirac and G. Rempe for comments and support. We thank D. Moehring for reading and improving the manuscript.

Author information

Reprints and permission information is available online at http://npg.nature.com/reprintsandpermissions. Correspondence and requests for materials should be addressed to T.S. 